Algebraic $\& \mathcal{G}$ Geometric Topology

Volume 2 (2002) 257-284

Published: 16 April 2002

ATG

\title{
Foliations with few non-compact leaves
}

\author{
Elmar Vogt
}

\begin{abstract}
Let $\mathcal{F}$ be a foliation of codimension 2 on a compact manifold with at least one non-compact leaf. We show that then $\mathcal{F}$ must contain uncountably many non-compact leaves. We prove the same statement for oriented $p$-dimensional foliations of arbitrary codimension if there exists a closed $p$ form which evaluates positively on every compact leaf. For foliations of codimension 1 on compact manifolds it is known that the union of all non-compact leaves is an open set [Hae].
\end{abstract}

\section{AMS Classification 57R30}

Keywords Non-compact leaves, Seifert fibration, Epstein hierarchy, foliation cycle, suspension foliation

\section{Introduction}

Consider a $C^{r}$-foliation $\mathcal{F}$ on a compact manifold with at least one non-compact leaf. Is it possible that this leaf is the only non-compact leaf of $\mathcal{F}$ ? If not, is it possible that there are only finitely many non-compact leaves, or countably many of them? Or must there always be uncountably many non-compact leaves? Do the answers depend on $r$ ? These questions were asked by Steve Hurder in [L], Problem A.3.1.

At first it seems obvious that for a foliation on a compact manifold the union of all non-compact leaves, if not empty, should have a non-empty interior. In fact, in codimension 1, and apart from flows, these are the foliations that come first to mind, this set is open. In [Hae], p.386, A. Haefliger proves that the union of all closed leaves of a codimension 1 foliation of a manifold with finite first mod 2 Betti number is a closed set. Therefore, the union of all non-compact leaves of a codimension 1 foliation of a compact manifold is open. Consequently, the set of all non-compact leaves of a codimension 1 foliation on a compact manifold is either empty or is uncountable.

But for foliations of codimension greater than 1 it is easy to construct examples on closed manifolds with the closure $C$ of the union of all non-compact leaves a 
submanifold of positive codimension. In fact, given $0<p<n$, there exist real analytic $p$-dimensional foliations on closed $n$-manifolds where $C$ is a $(p+1)$ dimensional submanifold. Therefore, the dimension of the closure of the union of all non-compact leaves can be quite small when compared to the dimension of the manifold, even for real analytic foliations (Compare this with Problem A.3.2 in $[\mathrm{L}]$ where a related question is asked for $C^{1}$-foliations). These examples are fairly straight forward generalizations of a construction of G. Reeb, [R],(A,III,c), and will be presented in Section 1 (Proposition 1.1).

The main results of this note extend the statement that for codimension 1 foliations on compact manifolds the set of non-compact leaves is either empty or uncountable in two directions. First we show that it also holds for foliations of codimension 2. The second result states that it is true in general if an additional homological condition is satisfied. To be more explicit, we first recall that a Seifert fibration is a foliation whose leaves are all compact and all have finite holonomy groups. Then we have:

Theorem 1 Let $\mathcal{F}$ be a foliation of codimension 2 on a compact manifold. Then $\mathcal{F}$ is either a Seifert fibration or it has uncountably many non-compact leaves.

Theorem 2 Let $\mathcal{F}$ be an oriented $C^{1}$-foliation of dimension $p$ on a compact manifold $M$. Assume that there exists a closed $p$-form $\omega$ on $M$ such that $\int_{L} \omega>0$ for every compact leaf $L$ of $\mathcal{F}$. Then $\mathcal{F}$ is either a Seifert fibration or $\mathcal{F}$ has uncountably many non-compact leaves.

Note that for a foliation on a manifold with boundary we will always assume that the boundary is a union of leaves.

The two theorems are corollaries of farther reaching but more technical results stated further down in this introduction as theorems $1^{\prime}$ and $2^{\prime}$.

We also include a short proof of the probably well known fact that for an arbitrary suspension foliation (i. e. a foliated bundle) over a compact manifold the set of non-compact leaves is empty or uncountable (Proposition 1.4).

As the statements of the two theorems indicate the techniques for their proofs are strongly related to the methods in [EMS] (and [Vo 1] for the codimension-2 case). There it was shown that foliations with all leaves compact on compact manifolds are Seifert fibrations if the homological condition of theorem 2 holds or if the codimension is 2 . The methods for the codimension 2 case are essentially due to D.B.A. Epstein who proved the corresponding result for circle 
foliations of compact 3-manifolds in [Ep 1]. In these papers the result for foliations with all leaves compact and of codimension 2 follows from the following more technical statement. Let $B_{1}$ be the union of leaves with infinite holonomy. For a foliation with all leaves compact of codimension 2 this set is empty if it is compact. This result in turn is obtained by constructing a compact transverse 2-manifold $T$ intersecting each leaf of $B_{1}$ but with $\partial T \cap B_{1}=\emptyset$. One then uses a generalization of a theorem of Weaver [Wea] to show that there is a compact neighborhood $N$ of $B_{1}$ and an integer $n$ such that all but finitely many leaves of $N$ intersect $T$ in exactly $n$ points. Thus all holonomy groups of leaves in $N$ are finite and $B_{1}=\emptyset$. The construction of $T$ is by downward induction, constructing transverse manifolds for a whole hierarchy $B_{\alpha}, \alpha$ an ordinal, of so-called bad sets. Here, given $B_{\alpha}$, the set $B_{\alpha+1}$ is defined as the union of all leaves of $B_{\alpha}$ with infinite holonomy group when the foliation is restricted to $B_{\alpha}$. (In [Ep 1], [EMS] and[Vo 1] a finer hierarchy of bad sets is used. There a leaf $L$ of $B_{\alpha}$ belongs to $B_{\alpha+1}$ if the holonomy group of $L$ of the foliation restricted to $B_{\alpha}$ is not trivial. The hierarchy we use in the present paper is called the coarse Epstein hierarchy in [EMS].)

For arbitrary codimension and still all leaves compact the ideas are due to R. Edwards, K. Millett, and D. Sullivan [EMS]. They show that $B_{1}$ is already empty if it is compact and if there exists a closed form $\omega$, defined in a neighborhood of $B_{1}$, satisfying $\int_{L} \omega>0$ for every leaf $L$ of $B_{1}$. The key idea in their proof is to construct a sequence of homologous leaves in the complement of $B_{1}$ converging to $B_{1}$ such that the volume of the leaves grows to infinity as the leaves approach $B_{1}$. Such a family gives rise to a non-trivial foliation cycle which is roughly the limit of the leaves each divided by some normalization factor which tends to infinity as the volume of the leaves goes to infinity. Therefore this cycle evaluates on $\omega$ to 0 since integration of $\omega$ is constant on the homologous leaves. On the other hand, if $B_{1}$ is non-empty, the cycle cannot evaluate to 0 on $\omega$ since it is non-trivial with support in $B_{1}$ and $\omega$ is positive when integrated over any leaf of $B_{1}$. (For a more detailed overview of this proof and an exposition of its main ideas read the beautifully written introduction of $[\mathrm{EMS}])$.

In our situation we first extend the notion of the hierarchy of bad sets to incorporate the occurrence of non-compact leaves. The points where the volume-of-leaf function with respect to some Riemannian metric is locally unbounded, used in the papers mentioned above for the definition of the first bad set $B_{1}$, is obviously inadequate. Also the union of all leaves with infinite holonomy misses some irregularities caused for example by simply connected non-compact leaves. Instead our criterion in the inductive definition of the hierarchy of bad sets puts 
a leaf of the bad set $B_{\alpha}$ in the next bad set $B_{\alpha+1}$ if for any transversal through this leaf the number of intersection points with leaves of $B_{\alpha}$ is not bounded. We begin with the whole manifold as $B_{0}$. In the presence of non-compact leaves the first bad set $B_{1}$ is not empty if the manifold is compact.

As opposed to the case when all leaves are compact, where the hierarchy of bad sets eventually reaches the empty set, it is now possible that the hierarchy stabilizes at a non-empty bad set $B_{\alpha}$, i. e. $B_{\alpha}=B_{\alpha+1}$ (and consequently $B_{\alpha}=B_{\beta}$ for all $\beta>\alpha$ ) and $B_{\alpha} \neq \emptyset$. But this will imply that $B_{\alpha}$ contains uncountably many non-compact leaves (actually a bit more can be said, see Proposition 3.5). Thus, we may assume that the hierarchy reaches the empty set. Then, in the codimension- 2 case, we manage to mimic all the steps in the construction of the transverse $2-$ manifold $T$ mentioned above, if the following condition is satisfied: let $N_{\alpha}$ be the union of the non-compact leaves in $B_{\alpha}$ 冫 $B_{\alpha+1}$; then $\operatorname{dim} N_{\alpha} \leq \operatorname{dim} \mathcal{F}$. Thus, and by some (further) generalization of Weaver's theorem we obtain the following theorem.

Theorem $\mathbf{1}^{\prime}$ Let $\mathcal{F}$ be a foliation of codimension 2, let $B_{0} \supset B_{1} \supset \cdots$ be its Epstein hierarchy of bad sets, and let $N_{\alpha}$ be the union of the non-compact leaves of $B_{\alpha} \backslash B_{\alpha+1}$. If $B_{1} \cup N_{0}$ is compact, then at least one of the following statements holds:

(i) for some ordinal $\alpha$ we have $B_{\alpha}=B_{\alpha+1}$ and $B_{\alpha} \neq \varnothing$-in this case no leaf of $B_{\alpha}$ is isolated (i. e. for no transverse open 2-manifold $T$ the set $T \cap B_{\alpha}$ contains an isolated point) and $B_{\alpha}$ contains a dense $G_{\delta}$ consisting of (necessarily uncountably many) non-compact leaves; furthermore, $\operatorname{dim} B_{\alpha}>\operatorname{dim} \mathcal{F}$, if all leaves of $B_{\alpha}$ are non-compact -, or

(ii) for some $\alpha \operatorname{dim} N_{\alpha}>\operatorname{dim} \mathcal{F}$, or

(iii) $\mathcal{F}$ is a Seifert fibration.

Theorem 1 follows from this since for any foliation the set $B_{1} \cup N_{0}$ is closed.

That, in a way, Theorem $1^{\prime}$ is best possible is shown by the examples of Section 1 mentioned above. They contain examples of real analytic foliations of codimension 2 on closed manifolds of any given dimension greater than 2 such that $B_{1}$ consists of finitely many compact leaves, and the dimension of the union of the non-compact leaves exceeds the leaf dimension by one.

The procedure for the proof of Theorem 2 is also similar to the proof in the case where all leaves are assumed to be compact. The sequence of homologous compact leaves for the definition of the foliation cycle is now assumed to converge to $B_{1} \cup N_{0}$ where as above $N_{0}$ is the union of all non-compact leaves in the 
complement of $B_{1}$, and the closed form $\omega$ has to be defined in a neighborhood of the closed set $B_{1} \cup N_{0}$. The construction of this sequence of leaves is to a certain degree easier in the presence of (not too many) non-compact leaves. Also the support of the limiting foliation cycle will essentially be disjoint from the non-compact leaves and thus they play no role in the evaluation of this cycle on $\omega$. More precisely, we have the following theorem.

Theorem $2^{\prime} \quad$ Let $\mathcal{F}$ be an oriented $C^{1}$-foliation of dimension $p$ on a manifold $M$, let $M=B_{0} \supset B_{1} \supset \cdots$ be its Epstein hierarchy of bad sets, and let $N_{0}$ be the union of the non-compact leaves in the complement of $B_{1}$. Assume that there exists a closed $p$-form $\omega$ defined in a neighborhood of $B_{1} \cup N_{0}$ such that $\int_{L} \omega>0$ for every compact leaf $L$ of $B_{1}$, and assume that $B_{1} \cup N_{0}$ is compact. Then at least one of the following statements holds:

(i) $\bigcap_{\alpha} B_{\alpha} \neq \emptyset$ - in this case no leaf of $\bigcap_{\alpha} B_{\alpha}$ is isolated and $\bigcap_{\alpha} B_{\alpha}$ contains a dense $G_{\delta}$-set consisting of (necessarily uncountably many) noncompact leaves -, or

(ii) $N_{0}$ is a non-empty open subset of $M$, in fact a non-empty union of components of the open set $M \backslash B_{1}$, or

(iii) $\mathcal{F}$ is a Seifert fibration.

In all the examples that I am aware of where a $p$-dimensional foliation on a compact manifold contains non-compact leaves, the union of all non-compact leaves is at least $p+1$ dimensional. Therefore, it would be interesting to know whether statement (i) in the two theorems above could be improved to: $B_{1}$ contains a subset of dimension greater than the leaf dimension consisting of non-compact leaves. I also do not know whether in codimension $\geq 3$ there are foliations on compact manifolds with at least 1 and at most countably many non-compact leaves.

Theorems 1 and $1^{\prime}$ hold for topological foliations, but we give a detailed proof only for the $C^{1}$-case, indicating the necessary changes for the topological case briefly at the end of section 4 . The proof in the $C^{0}$-case depends heavily on the intricate results of D.B.A. Epstein in [Ep 3].

Section 1 contains the examples mentioned above of real analytic foliations of codimension $q, q \geq 1$, on closed manifolds such that the closure of the union of all non-compact leaves is a submanifold of codimension $q-1$.

If one is content with $C^{r}$-foliations, $0 \leq r \leq \infty$, then one can construct such examples on many manifolds: given $p, k, n$ with $0<p<k \leq n$ and $p \leq n-2$ then any $n$-manifold which admits a $p$-dimensional $C^{r}$-foliation with all leaves 
compact will support also a $p$-dimensional $C^{r}$-foliation such that the closure of the union of all non-compact leaves is a non-empty submanifold of dimension $k$ (Proposition 1.2).

In addition, we give in Section 1 a simple proof of the well-known fact that for suspension foliations over compact manifolds, i. e. foliated bundles with compact base manifolds, the existence of one non-compact leaf implies the existence of uncountably many. This is an easy application of a generalization, due to D.B.A. Epstein [Ep 2], of a theorem of Montgomery [M].

In Section 2 we introduce some notation and gather a few results concerning the set of non-compact leaves of a foliation. In particular, we prove a mild generalization of the well known fact that the closure of a non-proper leaf of a foliation contains uncountably many non-compact leaves.

Section 3 introduces the notion of Epstein hierarchy of bad sets in the presence of non-compact leaves and we prove some of its properties. Section 4 contains the proof of Theorem $1^{\prime}$ along the lines indicated above. Finally, in Section 5 we construct the sequence of compact leaves approaching $B_{1} \cup N_{0}$ and the associated limiting foliation cycle, and establish the properties of this cycle to obtain the proof of Theorem $2^{\prime}$.

I have tried to make this paper reasonably self contained. But in Section 4 referring to some passages in [Vo 2] will be necessary for understanding the proofs in all details. The same holds for Section 5 where familiarity with [EMS] will be very helpful. I will give precise references wherever they are needed. Also, I will use freely some of the notions and results of the basic paper [Ep 2] on foliations with all leaves compact.

It will also be of help to visualize some of the examples of Section 1. Although they are simple they illustrate some of the concepts introduced later in the paper, and they give an indication of the possibilities expressed in Theorems $1^{\prime}$ and $2^{\prime}$ above.

This paper replaces an earlier preprint of the author with the same title. There the main result of [Vo 3] and calculations of the Alexander cohomology of the closure of the union of all non-compact leaves was used to prove a special case of Theorem 1 for certain 1-dimensional foliations on compact 3-manifolds.

In this paper finite numbers are also considered to be countable. 


\section{Foliations having a set of non-compact leaves of small dimension}

We generalize (in a trivial way) an example given by G. Reeb in $[R],(A, I I I, c)$. Let $F^{p}$ and $T^{k}$ be closed connected real analytic manifolds. Let $f: F^{p} \rightarrow \mathbb{R}$ be real analytic with 0 a regular value in the range of $f$, and let $g: T^{k} \rightarrow \mathbb{R}$ be real analytic with a unique maximum in $x_{0} \in T^{k}$. For convenience, let $g\left(x_{0}\right)=1$.

Let $\theta \in \mathbb{R} \bmod 2 \pi$ be coordinates for $S^{1}$ and consider for $x \in T^{k}$ the 1-form

$$
\omega(x)=\left((g(x)-1)^{2}+f^{2}\right) d \theta+g(x) d f
$$

on $F^{p} \times S^{1}$. One immediately checks that $\omega(x)$ is nowhere 0 and completely integrable. It thus defines a real analytic foliation $\mathcal{F}(x)$ of codimension 1 on $\{x\} \times F^{p} \times S^{1}$. These foliations fit real analytically together to form a foliation $\mathcal{F}$ of codimension $q=k+1$ on $T^{k} \times F^{p} \times S^{1}$. It is easy to describe the leaves of $\mathcal{F}(x)$. There are two cases.

Case $1 x \neq x_{0}$. Then $(g(x)-1)^{2}>0$, and $\omega(x)=0$ if and only if $d \theta=-\frac{g(x)}{(g(x)-1)^{2}+f^{2}} d f$. Therefore the leaves of $\mathcal{F}(x)$ are the graphs of the functions $h\left(\theta_{0}\right): F^{p} \rightarrow S^{1}$, given by

$$
h\left(\theta_{0}\right)(y)=\frac{-g(x)}{g(x)-1} \cdot \arctan \left(\frac{f(y)}{g(x)-1}\right)+\theta_{0}, 0 \leq \theta_{0}<2 \pi .
$$

These leaves are all diffeomorphic to $F^{p}$ and therefore compact.

Case $2 x=x_{0}$. Then $g(x)=1$, and $\omega\left(x_{0}\right)=f^{2} d \theta+d f$. We obtain two kinds of leaves for $\mathcal{F}\left(x_{0}\right)$. Let $F_{0}=f^{-1}(0)$. Then $\omega\left(x_{0}\right)=d f$ on $\left\{x_{0}\right\} \times F_{0} \times S^{1}$. Therefore, $\omega\left(x_{0}\right)=0$ implies $f=$ const, and the components of $\left\{x_{0}\right\} \times F_{0} \times S^{1}$ are compact leaves of $\mathcal{F}\left(x_{0}\right)$. In the complement of $\left\{x_{0}\right\} \times F_{0} \times S^{1}$ the foliation $\mathcal{F}\left(x_{0}\right)$ is given by $d \theta=-\frac{1}{f^{2}} d f$. Therefore, the leaves are components of the graphs of $k\left(\theta_{0}\right): F \backslash F_{0} \rightarrow S^{1}$, given by

$$
k\left(\theta_{0}\right)(y)=\frac{1}{f(y)}+\theta_{0}, 0 \leq \theta_{0}<2 \pi .
$$

Since $F$ is connected, no component of $F-F_{0}$ is compact. Thus $\left\{x_{0}\right\} \times(F \backslash$ $\left.F_{0}\right) \times S^{1}$ is the union of the non-compact leaves of $\mathcal{F}$, and we have the following result.

Proposition 1.1 Let $F^{p}$ and $T^{k}$ be real analytic closed manifolds of dimension $p>0$ and $k$ respectively. Then there exists a real analytic $p$-dimensional foliation $\mathcal{F}$ on $T^{k} \times F^{p} \times S^{1}$ such that the closure of the union of the noncompact leaves of $\mathcal{F}$ equals $\left\{x_{0}\right\} \times F^{p} \times S^{1}$ for some point $x_{0} \in T^{k}$. 
The construction above is quite flexible, especially if one allows the foliations to be smooth. For example

Proposition 1.2 Let $0 \leq r \leq \infty$ and let $M$ be an $n$-manifold which supports a $p$-dimensional $C^{r}$-foliation with all leaves compact, where $0<p \leq n-$ 2. Then for any integer $k$ with $p<k \leq n$ the manifold $M$ supports a $p$ dimensional $C^{r}$-foliation with the following property: the closure of the union of non-compact leaves is a non-empty submanifold of dimension $k$.

Proof The leaves with trivial holonomy form an open dense subset of any foliation with all leaves compact. Let $F$ be a leaf with trivial holonomy and $U$ a saturated neighborhood of $F$ of the form $F \times D^{n-p}$, foliated by $F \times\{y\}$, $y \in D^{n-p}$, where $D^{n-p}$ is the unit $(n-p)$-ball. Let $S^{1} \times D^{n-p-1} \hookrightarrow D^{n-p}$ be a smooth embedding into the interior of $D^{n-p}$ and let $K$ be a compact submanifold of the interior of $D^{n-p-1}$ of dimension $k-p-1$.

Let $f: F \rightarrow \mathbb{R}$ be smooth with 0 a regular value in the range of $f$, and let $h: D^{n-p-1} \rightarrow[0,1]$ be smooth with the following properties: $h$ and all its derivatives vanish on $\partial D^{n-p-1}, h(K)=1$, and $h(z)<1$ for all $z \in$ $D^{n-p-1} \backslash K$.

Replace on $F \times S^{1} \times D^{n-p-1}$ the product foliation induced from $F \times D^{n-p}$ by the smooth foliation defined on $F \times S^{1} \times\{z\}, z \in D^{n-p-1}$, by the 1 -form

$$
\omega(z)=\left((h(z)-1)^{2}+f^{2}\right) d \theta+h(z) d f .
$$

Then, as in our first example, the foliations of codimension 1 on $F \times S^{1} \times\{z\}$ defined by $\omega(z)=0$ fit smoothly together to form a foliation of $F \times S^{1} \times D^{n-p-1}$. On the boundary $F \times S^{1} \times \partial D^{n-p-1}$ this foliation fits smoothly to the product foliation on $F \times\left(D^{n-p} \backslash\left(S^{1} \times D^{n-p-1}\right)\right)$. The leaves in $F \times S^{1} \times\left(D^{n-p-1} \backslash K\right)$ are all diffeomorphic to $F$. Furthermore, $\left(F \backslash f^{-1}(0)\right) \times S^{1} \times K$ is a union of non-compact leaves, each one of which is diffeomorphic to some component of $F \backslash f^{-1}(0)$. Therefore $F \times S^{1} \times K$ is the closure of the union of the non-compact leaves.

Proposition 1.2 suggests the following question.

Question 1.3 Does there exist a foliation of dimension $p$ on a compact manifold $M$ such that the closure of the union of all non-compact leaves is nonempty and has dimension $p$ ? 
By the result of Haefliger mentioned in the introduction [Hae], page 386, such a foliation has codimension at least 2 , and in the case of codimension 2 , there must, by Theorem $1^{\prime}$, be an $\alpha$ such that $B_{\alpha}=B_{\alpha+1} \neq \emptyset$, and $B_{\alpha}$ contains compact leaves. Furthermore, in general, it cannot be a suspension foliation. A suspension foliation $\mathcal{F}_{\varphi}$ is given by a homomorphism $\varphi: \pi_{1}(B) \rightarrow \operatorname{Homeo}(T)$, where $B$ and $T$ are manifolds and $B$ is connected. One foliates $\tilde{B} \times T$ by $\tilde{B} \times\{t\}, t \in T$, where $\tilde{B}$ is the universal cover of $B$. This foliation is invariant under the obvious action of $\pi_{1}(B)$ and induces the foliation $\mathcal{F}_{\varphi}$ on the quotient $\tilde{B} \times T / \varphi$ by this action.

Proposition 1.4 Let $B$ and $T$ be manifolds with $B$ closed and connected, let $\varphi: \pi_{1} B \rightarrow \operatorname{Homeo}(T)$ be a homomorphism and assume that the associated suspension foliation $\mathcal{F}_{\varphi}$ has non-compact leaves. Let $N$ be the closure of the union of the non-compact leaves, and let $W$ be a component of $(\tilde{B} \times T / \varphi) \backslash$ $N$. Then the closure of $W$ consists of compact leaves. In particular, $N$ does not contain any isolated leaf, and $(\tilde{B} \times T / \varphi) \backslash N$ consists of infinitely many components unless $N$ contains interior points. Furthermore, the dimension of $N$ is at least equal to $(\operatorname{dim} B+\operatorname{dim} T-1)$. (As definition of dimension we may take any of the notions of covering dimension, inductive dimension, or cohomological dimension, which are equivalent in our situation).

If $\operatorname{dim} T=1$, then the union of all non-compact leaves is open.

Proof The quotient space $M=\tilde{B} \times T / \varphi$ is a fibre bundle with fibre $T$. The fibres are transverse to the foliation $\mathcal{F}_{\varphi}$. Compact leaves of $\mathcal{F}_{\varphi}$ correspond to finite orbits of the group $G=\varphi\left(\pi_{1}(B)\right) \subset \operatorname{Homeo}(T)$. We identify $T$ with the fibre over the basepoint of $B$. Let $W$ be a component of $M \backslash N$ and let $x$ be a point of $W \cap T$. Let $G_{O x}$ be the normal subgroup of finite index of $G$ whose elements keep the orbit of $x$ pointwise fixed. Let $W_{x}$ be the component of $W \cap T$ containing $x$ and let $G_{W}$ be be the restriction of $G_{O x}$ to $W_{x}$ considered as a subgroup of Homeo $\left(W_{x}\right)$. Every orbit of $G_{W}$ is finite and $W_{x}$ is a connected manifold. Then, by Theorem 7.3 in [Ep 2], an extension of the main result in $[\mathrm{M}]$ to groups of homeomorphisms, $G_{W}$ is finite, say of order $g$. This implies that the $G_{O x}$-orbit of any point in the closure of $W_{x}$ contains at most $g$ points. Thus any orbit in the closure of $W \cap T$ under the action of $G$ is finite, and all leaves in the closure of $W$ must be compact.

Since this is true for any component $W$ of $M \backslash N$ any neighborhood of a point in $N$ which is not a point of the closure of int $(N)$ must intersect infinitely many components of $M \backslash N$. In particular, $\operatorname{dim} N \geq \operatorname{dim} M-1=\operatorname{dim} B+\operatorname{dim} T-1$. 
If $\operatorname{dim} T=1$, we first reduce our problem to the case where $T$ is connected. To do this we observe that $T$ decomposes naturally into disjoint subspaces each of which is a union of components of $T$ on which $G$ acts transitively. So we may assume that $G$ acts transitively on the components of $T$. If then the number of components is infinite, then all leaves are non-compact. So we may assume that the number of components of $T$ is finite. We then replace $G$ by the subgroup of finite index whose elements preserve every component. This corresponds to passing to a finite covering of $M$ with the induced foliation. On each component of this covering space the induced foliation is a suspension with one component of $T$ as fibre. So we may assume that $T$ is connected, i.e. $T$ is either $\mathbb{R}$ or $S^{1}$. We may furthermore assume that all elements of $G$ are orientation preserving.

If $T=\mathbb{R}$, then every finite orbit is a global fixed point. Therefore, the union of all finite orbits is closed, and we are done. If $T=S^{1}$ either all leaves are non-compact or we may pass to the subgroup of finite index keeping a finite orbit pointwise fixed. For this subgroup every finite orbit is again a global fixed point, and we can argue as before.

Corollary 1.5 (Well known) Let $\mathcal{F}_{\varphi}$ be the suspension foliation associated to the homomorphism $\varphi: \pi_{1}(B) \rightarrow \operatorname{Homeo}(T)$ and assume that $B$ is closed. Then $\mathcal{F}_{\varphi}$ contains uncountably many non-compact leaves or none. If $\operatorname{dim} T=$ 1 , the union of all non-compact leaves is open.

Proof Assume that $\mathcal{F}_{\varphi}$ contains a non-compact leaf, and let $N$ be the closure of the union of the non-compact leaves of $\mathcal{F}_{\varphi}$. The set $N$ with its induced foliation is a foliated space in the sense of [EMT]. The main result of [EMT] implies that the union of all leaves of $N$ with trivial holonomy is a dense $G_{\delta}$ in $N$. By Proposition 1.4, $\operatorname{dim} N \geq \operatorname{dim} \mathcal{F}_{\varphi}+1$ and $N$ does not contain any isolated leaf. Therefore, the Baire category theorem implies that $N$ contains uncountably many leaves with trivial holonomy. Let $L$ be a compact leaf of $N$. Then every neighborhood of $L$ intersects a non-compact leaf (of $N$ ). Therefore, the holonomy of $L$ is non-trivial, i.e. the leaves of $N$ with trivial holonomy are all non-compact.

\section{Uncountably many versus isolated non-compact leaves}

The material in this short section is standard. We include it to fix and introduce notation. 
Let $\mathcal{F}$ be a foliation of a manifold $M$ and let $A \subset M$ be a union of leaves. We call a leaf $L \subset A$ isolated (with respect to $A$ ) if $L$ is an open subset of $C l(A)$. Recall that a leaf of a foliation is called proper if its leaf topology coincides with the induced topology as a subset of $M$. Obviously, any leaf which is isolated with respect to some $A$ is proper, and the proper leaves are exactly those leaves which are isolated with respect to themselves. We will denote the union of isolated leaves with respect to the saturated set $A$ by $I(A)$.

Proposition 2.1 Let $A$ be a union of non-compact leaves of a foliation. Then at least one of the following holds:

(i) $\mathrm{Cl}(A)$ contains uncountably many non-compact leaves, or

(ii) $A \subset C l(I(A))$.

In particular, the closure of a non-proper leaf contains uncountably many noncompact leaves.

Proof Assume that $B:=A \backslash C l(I(A))$ is not empty. Any isolated leaf with respect to $B$ is also isolated with respect to $A$. Therefore $I(B)=\emptyset$. Let $U=D^{\prime} \times D$ be a foliation chart with $D^{\prime}$ connected and tangent to the foliation and assume that $U \cap B \neq \emptyset$. We identify $D$ with $\{y\} \times D$ for some basepoint $y \in D^{\prime}$. Then $C:=C l(B) \cap D$ is a closed non-empty subset of $D$ which contains no isolated points. By the main theorem of [EMT] the union $H$ of leaves of $C l(B)$ with trivial holonomy is a dense $G_{\delta}$. Since all leaves in $B$ are non-compact, the compact leaves in $C l(B)$ all have non-trivial holonomy. Therefore all leaves in $H$ are non-compact. Since $H \cap C$ is a dense $G_{\delta}$ in $C$, the set $H \cap C$ is uncountable by the Baire category theorem. Every leaf of our foliation intersects $D$ in an at most countable set. Therefore the set of non-compact leaves of $C l(B)$ intersecting $D$ is uncountable.

\section{The Epstein hierarchy in the presence of non- compact leaves}

There are several possible ways to generalize the notion of Epstein hierarchy (see [Ep 1] or [Vo 2]) to foliations admitting non-compact leaves. For our purposes definition 3.4 below seems to be the best choice. Before we come to this we need some notation. 
Notation 3.1 Let $(M, \mathcal{F})$ be a codimension $k$ foliated manifold. A transverse manifold is a $k$-dimensional submanifold $T$ of $M$ which is transverse to $\mathcal{F}$ and whose closure $C l(T)$ is contained in the interior of a $k$-dimensional submanifold transverse to $\mathcal{F}$. A transverse manifold $T$ may or may not have a boundary, denoted by $\partial T$. We call $\operatorname{int} T:=T \backslash \partial T$ the interior of $T$, and call $T$ open, if $T=\operatorname{int} T$.

Notation 3.2 Let $T$ be a transverse manifold of a foliated manifold $(M, \mathcal{F})$. Then $\sec _{T}: M \longrightarrow \overline{\mathbb{N}}:=\mathbb{N} \cup\{\infty\}$ is the map which associates to $x \in M$ the cardinal of the set $T \cap L_{x}$, where $L_{x}$ is the leaf through $x$.

The topology on $\overline{\mathbb{N}}$ is the one point compactification of $\mathbb{N}=\{0,1,2, \ldots\}$. Then we have

Property 3.3 (a) $\sec _{T}$ is continuous in every point of $\sec _{(T \backslash \partial T)}^{-1}(\infty)$.

(b) If $L_{x} \cap \partial T=\emptyset$, then $\sec _{T}$ is lower semi-continuous in $x$.

The proofs are obvious.

Definition 3.4 Let $(M, \mathcal{F})$ be a foliated manifold. The Epstein hierarchy of bad sets of $\mathcal{F}$ is a familiy $\left\{B_{\alpha}=B_{\alpha}(\mathcal{F})\right\}$ of subsets of $M$ indexed by the ordinals and is defined by transfinite induction as follows:

$$
\begin{aligned}
& B_{0} \quad=M \quad ; \\
& B_{\alpha}=\bigcap_{\beta<\alpha} B_{\beta} \quad, \quad \text { if } \alpha \text { is a limit ordinal; } \\
& B_{\alpha+1}=\left\{x \in B_{\alpha} \quad: \quad \text { for every transverse manifold } T \text { with } x \in \operatorname{int} T\right. \\
& \left.\sup \left\{\sec _{T}(y): y \in B_{\alpha}\right\}=\infty\right\} \text {. }
\end{aligned}
$$

Obviously each $B_{\alpha}$ is a closed invariant set.

Proposition 3.5 (1) If $B_{\alpha+1}=B_{\alpha}$ and $B_{\alpha} \neq \emptyset$ then for any transverse open manifold $T$ with $T \cap B_{\alpha} \neq \emptyset$ we have

(i) $T \cap B_{\alpha}$ contains no isolated point and

(ii) $T \cap B_{\alpha}$ contains a dense (necessarily uncountable) $G_{\delta}$-set $R$ of points lying in non-compact leaves.

(2) If $B_{\alpha+1}$ contains at most countably many non-compact leaves, then $B_{\alpha+1}$ is nowhere dense in $B_{\alpha}$. 
Proof By the Baire category theorem a locally compact space without isolated points does not contain a countable dense $G_{\delta}$-set. For any transverse open manifold $T$ the set $T \cap B_{\alpha}$ is locally compact, and its isolated points belong to $B_{\alpha} \backslash B_{\alpha+1}$. Any leaf intersects any transverse manifold in an at most countable set. Therefore it suffices to show the following. For every open transverse manifold $T$ with $T \cap B_{\alpha} \neq \emptyset$ and $T \cap B_{\alpha} \subset B_{\alpha+1}$ the set $T \cap B_{\alpha}$ satisfies properties (i) and (ii).

Let $\sec _{\alpha}$ be the restriction of $\sec _{T}$ to the locally compact space $T \cap B_{\alpha}$. By (3.3) and again the Baire category theorem $T \cap B_{\alpha}$ contains a dense $G_{\delta}$-set $R$ of points where $\sec _{\alpha}$ is continuous. Assume that there exists a point $y \in$ $R \cap \sec _{\alpha}^{-1}(\mathbb{N})$. Then $\sec _{\alpha}$ is constant in a neighborhood of $y$. This means that $y$ is a point in $B_{\alpha} \backslash B_{\alpha+1}$, which is not possible. Therefore, $R \subset \sec _{\alpha}^{-1}\{\infty\}$. This implies that every point of $R$ is contained in a non-compact leaf of $B_{\alpha+1}$.

The first claim of the next proposition is due to the convention that manifolds are second countable.

Proposition 3.6 If $B_{1} \neq B_{0}$, let $\gamma:=\min \left\{\beta \mid B_{\beta+1}=B_{\beta+2}\right\}$. Otherwise, let $\gamma$ be 0 . Then the following holds:

(i) $\gamma$ is a countable ordinal;

(ii) if $M \neq \emptyset$ and $B_{\alpha}$ is compact for some $\alpha<\gamma$ then $B_{\gamma} \neq \emptyset$;

(iii) if $B_{\gamma}$ is compact then all leaves in $B_{\gamma}$ are compact.

The last statement is due to the fact that $B_{\beta+1} \neq \emptyset$ if $B_{\beta}$ is compact and contains a non-compact leaf.

For further reference we note the following proposition.

Proposition 3.7 Each point of the interior of $B_{1}$ is contained in $\bigcap_{\alpha} B_{\alpha}$.

Proof Let $x$ be an interior point of $B_{1}$, and let $T$ be an open transverse manifold with $x \in T \subset B_{1}$. Then $\sec _{T}=\sec _{T \cap B_{1}}$. Thus $T \subset B_{2}$, and, by transfinite induction, $T \subset B_{\alpha}$ for all $\alpha$. 


\section{Codimension 2 foliations}

For simplicity we assume that all foliations are $C^{1}$, but the main result (Theorem $1^{\prime}$ ) is also true for $C^{0}$-foliations. We will indicate the necessary changes in an appendix at the end of this section.

As in the case of the study of foliations with all leaves compact there are two ingredients which make the codimension 2 case special. The first one is the fact that for $\alpha \geq 1$ the bad set $B_{\alpha}$ is transversally of dimension at most 1 if $\operatorname{dim} \bigcap_{\alpha} B_{\alpha}<\operatorname{dim} B_{0}$ (Proposition 3.7). The second one is a generalization of Weaver's Lemma [Wea] which takes in our setting the following form.

Proposition 4.1 Let $\mathcal{F}$ be a foliation of codimension 2 and $T$ a transverse 2-manifold. Let $C \subset T$ be compact connected and $W$ be the union of all leaves through points of $C$. Let $E \subset C$ be the set of points of $C$ lying in a non-compact leaf. We assume that

(i) no compact leaf of $W$ intersects $\partial T$,

(ii) every non-compact leaf of $W$ intersects $T$ in infinitely many points,

(iii) for any loop $\omega$ of a compact leaf through a point $x \in C$ a representative of the associated holonomy map defined in a neighborhood of $x$ in $T$ preserves the local orientation, and

(iv) $E$ is a countable union of disjoint closed sets $E_{j}$.

Then either all leaves of $W$ are non-compact, or there exists an integer $\rho$ such that all but finitely many leaves of $W$ intersect $T$ in exactly $\rho$ points. In the latter case the finitely many other leaves of $W$ intersect $T$ in fewer than $\rho$ points.

Proof For each positive integer $m$ let

$$
C_{m}=\left\{x \in C: \sec _{T}(x) \leq m\right\},
$$

and let $D_{m} \subset C_{m}$ be the set of non-isolated points of $C_{m}$. Clearly each $C_{m}$ and $D_{m}$ is closed, each $C_{m} \backslash D_{m}$ is at most countable, and we have a decomposition

$$
C=\bigcup_{m \geq 1}\left(D_{m} \backslash D_{m-1}\right) \cup\{\text { countable set }\} \cup \bigcup_{j} E_{j}
$$

into a countable union of disjoint sets. We claim that each $D_{m} \backslash D_{m-1}$ is closed. For if not, then there exists $x \in D_{m-1}$ with $x \in C l\left(D_{m} \backslash D_{m-1}\right)$. By hypothesis (ii) the leaf $L_{x}$ through $x$ is compact. Now we can argue as in 
the proof of Lemma 3.4 in [Vo 2] to obtain a representative $\mathrm{h}$ of an element of the holonomy group of $L_{x}$ such that $d h(x)$ has a non-zero fixed vector $v$ and a periodic vector $w$ with least period $\nu>1$. But this contradicts hypothesis (iii). (Hypothesis (i) is needed for imitating the proof of 3.4 in [Vo 2].)

Since by hypothesis (iv) also the $E_{j}$ are closed, the compact connected set $C$ is a countable disjoint union of closed sets. Then a theorem of Sierpinski $[\mathrm{Ku}]$, $\S 47$ III Theorem 6 , states that $C$ must be equal to one of the sets of which it is the disjoint union. So either $C$ is one of the $E_{j}$ and all leaves of $W$ are non-compact, or $C$ is a single point in a compact leaf, or there exists $\rho$ with $C=D_{\rho} \backslash D_{\rho-1}$. The set of points of $D_{\rho} \backslash D_{\rho-1}$ which lie in leaves intersecting $T$ in less than $\rho$ points is $\left(D_{\rho} \backslash D_{\rho-1}\right) \cap C_{\rho-1}$. But this set is compact and discrete and therefore finite.

An easy consequence of 4.1 is the following result.

Proposition 4.2 Let $\mathcal{F}$ be a codimension 2 foliation, $B_{0} \supset B_{1} \supset \cdots$ the Epstein hierarchy of $\mathcal{F}$, and $N_{\alpha}$ the union of all non-compact leaves of $\mathcal{F}$ in $B_{\alpha} \backslash B_{\alpha+1}$. Assume that the closure of every leaf of $\mathcal{F}$ is compact, that $B_{1}$ is a non-empty set, and that $\operatorname{dim} N_{\alpha} \leq \operatorname{dim} \mathcal{F}$ for every $\alpha \geq 0$. Furthermore assume that $\bigcap_{\alpha} B_{\alpha}$ is empty or contains only non-compact leaves and that $\operatorname{dim} \bigcap_{\alpha} B_{\alpha}<\operatorname{dim} B_{0}$. Then there does not exist a compact transverse 2-manifold $T$ intersecting each leaf of $B_{1}$ and with $\partial T \cap B_{1}=\emptyset$.

Proof The proof is by contradiction. It is clear that we may assume that $\mathcal{F}$ is transversely orientable. We will show below (Lemma 4.3) that with our hypotheses we can always arrange $T$ so that $\partial T$ does not intersect any noncompact leaf. The union of non-compact leaves in $B_{0} \backslash B_{1}$ is closed in $B_{0} \backslash B_{1}$. Therefore we find a compact neighborhood $K$ of $\partial T$ in $T$ such that $K \cap B_{1}=\emptyset$ and every leaf through a point of $K$ is compact. This implies that the union $S_{K}$ of leaves through $K$ is compact and $\mathcal{F}$ restricted to $S_{K}$ is a Seifert fibration (Here we extend the notion of Seifert fibration to foliated sets. Such a set will be called a Seifert fibration if all leaves are compact with finite holonomy groups).

Now consider a component $D$ of $T \backslash S_{K}$ such that $D \cap B_{1} \neq \emptyset$ (such a component exists) and apply Proposition 4.1 to $C=\bar{D} \subset T \backslash \operatorname{Int}(K)$. Hypotheses (i), (ii) and (iii) of 4.1 are clearly satisfied, the last one because we have assumed $\mathcal{F}$ to be transversely orientable. The union $N_{\alpha}$ of all non-compact leaves in $B_{\alpha} \backslash B_{\alpha+1}$ is a closed subset of $B_{0} \backslash B_{\alpha+1}$ which intersects $T$ in a set of dimension 0 . Therefore, for each $\alpha$, the set $T \cap N_{\alpha}$ is a countable disjoint 
union of compact sets. By Proposition 3.6 there are only countably many nonempty $N_{\alpha}$ 's. Furthermore, if $\bigcap B_{\alpha}$ contains non-compact leaves, all leaves of $\bigcap B_{\alpha}$ are non-compact. Since $\bigcap B_{\alpha}$ is closed, also Condition (iv) is satisfied, and we are entitled to apply 4.1. Since $D$ is a non-empty open subset of $T$ and, by hypothesis, the union of all non-compact leaves has dimension less than the dimension of $B_{0}$, not all points of $\bar{D}$ lie in non-compact leaves. Consequently all leaves intersecting $\bar{D}$ are compact and the function $\sec _{T}$ is bounded on $\bar{D}$. This implies $B_{1} \cap D=\emptyset$, which is a contradiction.

The next (easy) lemma is true in by far more generality. We only state it for the case of interest to us.

Lemma 4.3 Let $T$ be a 2-manifold with compact boundary $\partial T$ and let $N \subset T$ be a 0-dimensional subset which is closed in a neighborhood of $\partial T$. Then for any neighborhood $U$ of $\partial T$ there exists a submanifold $T^{\prime} \subset T$ with compact boundary $\partial T^{\prime}$ such that $T \backslash U \subset T^{\prime}$ and $\partial T^{\prime} \cap N=\emptyset$.

Proof By looking at each component of $\partial T$ separately the lemma reduces to the statement that for any closed 0 -dimensional subset $N$ of $S^{1} \times[0,1]$ we find a neighborhood $K$ of $S^{1} \times\{0\}$ which is a compact 2-manifold with boundary $\partial K$ such that $S^{1} \times\{0\} \subset \partial K$ and $\partial K \cap N=\left(S^{1} \times\{0\}\right) \cap N$.

Since $N$ is 0-dimensional and closed, $N$ is for any $\epsilon>0$ a finite disjoint union of closed sets of diameter less than $\epsilon$, where we metrize $S^{1} \times[0,1]$ by considering it as a smooth submanifold of $R^{2}$. In particular, $N$ is the union of closed sets $N_{0}$ and $N_{1}$ with $\left(S^{1} \times\{0\} \cup N_{0}\right) \cap\left(S^{1} \times\{1\} \cup N_{1}\right)=\emptyset$. Let $d$ be the distance between $\left(S^{1} \times\{0\}\right) \cup N_{0}$ and $\left(S^{1} \times\{1\}\right) \cup N_{1}$. Then there exist finitely many closed disks $D_{1}, D_{2}, \ldots, D_{s}$ of radius $r<d$ such that $\left\{\operatorname{Int} D_{i}\right\}$ covers $\left.\left(S^{1} \times\{0\} \cup N_{0}\right)\right\}$, their boundaries $\left\{\partial D_{i}\right\}$ are in general position, and $D_{i} \cap N_{1}=\emptyset$ for all $i$. Then $K:=\bigcup_{i} D_{i} \cap\left(S^{1} \times[0,1]\right)$ is a 2-manifold with piecewise smooth boundary having the desired properties. We may, if we want to, smooth $\partial K$. Taking the component containing $S^{1} \times\{0\}$ and filling in some components of $\partial K$ bounding 2-cells in $S^{1} \times[0,1]$ we may also assume that $K$ is an annulus.

The final step in the proof of Theorem $1^{\prime}$ is the next proposition.

Proposition 4.4 Let $\mathcal{F}$ be an orientable and transversely orientable foliation of codimension 2, $B_{0} \supset B_{1} \supset \cdots$ its Epstein hierarchy, and $N_{\alpha}$ the union of all non-compact leaves of $\mathcal{F}$ in $B_{\alpha} \backslash B_{\alpha+1}$. Assume that $B_{1}$ is compact, that 
$\bigcap_{\alpha} B_{\alpha}$ is empty or consists of non-compact leaves only, and that $\bigcap_{\alpha} B_{\alpha}$ and all $N_{\alpha}$ have dimension at most equal to $\operatorname{dim} \mathcal{F}$. Then there exists a compact transverse 2-manifold $T$ intersecting every leaf of $B_{1}$ such that $\partial T \cap B_{1}=\emptyset$.

Proof In the absence of non-compact leaves (when $\bigcap_{\alpha} B_{\alpha}$ and all $N_{\alpha}$ are empty) the proposition was proved in [EMS] and [Vo 1] by extending the key ideas of Epstein in [Ep 1]. Our proof here is basically the same by noticing at each step that the non-compact leaves cause no additional difficulties.

Assume first that $\bigcap_{\alpha} B_{\alpha}=\emptyset$. Then by Proposition 3.6 there exists an ordinal $\gamma$ such that $B_{\gamma} \neq \emptyset$ and $B_{\gamma+1}=\emptyset$. We may assume that $\gamma \geq 1$, for otherwise there is nothing to prove. Then $B_{\gamma}$ is compact and again by 3.6 contains only compact leaves. Therefore $B_{\gamma}$ is a Seifert fibration with an at most 1dimensional leaf space. The techniques of [EMS] and [Vo 1] then show that there exists a compact transverse manifold $T_{\gamma}$ intersecting each leaf of $B_{\gamma}$ such that $\partial T_{\gamma} \cap B_{\gamma}=\emptyset$. For a detailed proof see [Vo 2], Proposition 4.7.

If $\bigcap_{\alpha} B_{\alpha} \neq \emptyset$ there exists an ordinal $\gamma$ such that $B_{\gamma}=\bigcap_{\alpha} B_{\alpha}$. By hypothesis $B_{\gamma}$ is transversely 0 -dimensional and thus we can again find a compact transverse $T_{\gamma}$ with the properties above.

The idea is now to use downward induction, i. e., if $\alpha>1$, and if $T_{\alpha}$ is a compact transverse 2-manifold which intersects every leaf of $B_{\alpha}$ and whose boundary $\partial T_{\alpha}$ is disjoint from $B_{\alpha}$, we have to construct for some $\beta<\alpha$ a transverse 2 -manifold $T_{\beta}$ having the same properties with respect to $B_{\beta}$. If $\alpha$ is a limit ordinal then for some $\beta<\alpha$ the 2-manifold $T_{\alpha}$ intersects every leaf of $B_{\beta}$ and $\partial T_{\alpha} \cap B_{\beta}=\emptyset$. This can be seen as follows.

The union $A$ of leaves of $\mathcal{F}$ not intersecting $\operatorname{int} T_{\alpha}$ is closed. Therefore, for any $\beta \geq 1$ the set $\left(A \cup \partial T_{\alpha}\right) \cap B_{\beta}$ is compact and $\bigcap_{\delta<\alpha}\left(\left(A \cup \partial T_{\alpha}\right) \cap B_{\delta}\right)=$ $\left(A \cup \partial T_{\alpha}\right) \cap B_{\alpha}=\emptyset$. It follows that for some $\beta<\alpha$ we have $\left(A \cup \partial T_{\alpha}\right) \cap B_{\beta}=\emptyset$ which implies that $T_{\alpha}$ intersects every leaf of $B_{\beta}$ and $B_{\beta} \cap \partial T_{\alpha}=\emptyset$.

So we may assume that $\alpha$ is not a limit ordinal. By Lemma 4.3 we may also assume that for the union $N_{\alpha-1}$ of all non-compact leaves of $B_{\alpha-1} \backslash B_{\alpha}$ we have $N_{\alpha-1} \cap \partial T_{\alpha}=\emptyset$.

Now, $B_{\alpha-1} \backslash\left(N_{\alpha-1} \cup B_{\alpha}\right)$ is a Seifert fibration. Since $\partial T_{\alpha}$ is compact we find a closed invariant neighborhood $K_{0}$ of $B_{\alpha} \cup N_{\alpha-1}$ in $B_{\alpha-1}$ such that $T_{\alpha}$ intersects every leaf of $K_{0}$ and $K_{0} \cap \partial T_{\alpha}=\emptyset$. Since exceptional leaves of foliated Seifert fibred subsets of a $C^{1}$-foliation are isolated ([Vo 2], Lemma 4.4) we may also assume that the set theoretic boundary $\operatorname{Fr}_{B_{\alpha-1}}\left(K_{0}\right)$ of $K_{0}$ in $B_{\alpha-1}$ does not 
contain any exceptional leaf of the Seifert fibration $\mathcal{F} \mid\left(B_{\alpha-1} \backslash\left(N_{\alpha-1} \cup B_{\alpha}\right)\right)$. (As a reminder: a leaf of a Seifert fibration is called exceptional, if its holonomy group is non-trivial.)

Below we will establish the following claim.

Claim 4.5 Let $E$ be the union of the exceptional leaves of the Seifert fibration $\mathcal{F} \mid\left(B_{\alpha-1} \backslash K_{0}\right)$. Then there exists a compact invariant neighborhood $N$ of $E$ in $B_{\alpha-1} \backslash K_{0}$ and a compact transverse manifold $S$ with the following properties

(i) $S$ intersects every leaf of $K_{1}=K_{0} \cup N$;

(ii) $\partial S \cap K_{1}=\emptyset$;

(iii) there exists a $\rho>0$ and an invariant neighborhood $U_{1}$ in $B_{\alpha-1}$ of the point set theoretic boundary $F_{B_{\alpha-1}} K_{1}$ of $K_{1}$ in $B_{\alpha-1}$ such that every leaf of $U_{1}$ intersects $S$ in exactly $\rho$ points.

Assuming that 4.5 is true we then proceed as in [Ep 1], [EMS], [Vo 1], [Vo 2] to extend $S$ to a transverse manifold having properties (i) and (ii) above with $K_{1}$ replaced by $B_{\alpha-1}$. The idea is to cover the locally trivial bundle $C l\left(B_{\alpha-1} \backslash K_{1}\right)$ by finitely many bundle charts $C_{2}, \ldots, C_{n}$ and then to construct inductively transverse compact manifolds $S_{1}=S, S_{2}, \ldots, S_{n}$ such that $S_{i}$ has properties (i), (ii), and (iii) above with $K_{1}$ replaced by $K_{i}=K_{1} \cup C_{2} \cup \cdots \cup C_{i}$. This is done by choosing for each $C_{i}$ a compact transverse manifold $D_{i}$ with $\partial D_{i} \cap C_{i}=\emptyset$ and intersecting each leaf of $C_{i}$ in exactly $\rho$ points. Then we shrink at each step $S_{i}$ and $D_{i+1}$ somewhat and adjust $D_{i+1}$ so that $S_{i+1}=S_{i} \cup D_{i+1}$ is a transverse 2-manifold having properties (i), (ii), and (iii) with regard to $K_{i+1}$. For a detailed description of this see [Vo 2], proof of 4.7 (Note that in figure 2 of [Vo 2] each $\Gamma$ should be interpreted as the intersection symbol $\cap$ ).

Proof of 4.5 (An adaptation of the proof in [Ep 1], Section 10, to our situation.) Since $K_{0} \cap \partial T_{\alpha}=\emptyset$, since $K_{0}$ is a neighborhood of $N_{\alpha-1} \cup B_{\alpha}$ in $B_{\alpha-1}$ and since $F_{B_{\alpha-1}}\left(K_{0}\right)$ does not contain an exceptional leaf of $B_{\alpha-1} \backslash\left(N_{\alpha-1} \cup B_{\alpha}\right)$, we find an invariant compact neighborhood $V=V_{1} \cup \cdots \cup V_{k}$ of $F r_{B_{\alpha-1}}\left(K_{0}\right)$ in $B_{\alpha-1}$ such that $V \cap E=\emptyset$, the $V_{i}$ are disjoint compact invariant sets and $\sec _{T_{\alpha}}$ restricted to each $V_{i}$ is constant with value, say $n_{i}$. We will assume that the $n_{i}$ are pairwise distinct. Let $U=U_{1} \cup \cdots \cup U_{k}$ be another compact invariant neighborhood of $\operatorname{Fr}_{B_{\alpha-1}}\left(K_{0}\right)$ such that for all $i$ we have $U_{i} \subset \operatorname{int}_{B_{\alpha-1}}\left(V_{i}\right)$. Then every component $C$ of $K_{0}$ which is not entirely contained in $V_{i}$ and meets $U_{i}$ has infinitely many leaves in $V_{i}$. Our hypotheses let us apply Proposition 4.1 to components of $K_{0} \cap T_{\alpha}$. From this we conclude that no component of $K_{0}$ 
will intersect two of the sets $U_{i}$. It is now a routine matter (see $[\mathrm{Ku}], \S 47$ II Theorem 3) to decompose $K_{0}$ into disjoint closed subsets $K_{0,1} \cup \cdots \cup K_{0, k}$ such that $K_{0, i} \cap U_{j}$ is empty for $i \neq j$. The closure of $B_{\alpha-1} \backslash K_{0}$ is compact, and $\mathcal{F}$ restricted to this set is a Seifert fibration. Therefore, $E$ is a finite union of leaves $L_{1}, \ldots, L_{m}$. Let $U_{k+i}$ be a compact invariant neighborhood of $L_{i}$ in $B_{\alpha-1}$. If the $U_{k+i}$ are small enough we may assume that all leaves of $U_{k+i}$ intersect a transverse disk $D_{i}$ in exactly $n_{k+i}$ points except $L_{i}$ which intersects $D_{i}$ once. We may further assume that $D_{i} \cap K_{0}=\emptyset, U_{k+i} \cap \partial D_{i}=\emptyset$ and that for $i \neq j U_{k+i} \cap D_{j}=\emptyset$. Now let $T_{i}, i=1, \ldots, k$, be a compact 2-manifold-neighborhood of $K_{0, i} \cap T_{\alpha}$ having the following properties

(i) $K_{0, i} \cap T_{j}=\emptyset \quad, i \neq j$;

(ii) $K_{0, i} \cap \partial T_{i}=\emptyset \quad, i=1, \ldots, k$;

(iii) $T_{i} \cap U_{k+j}=\emptyset \quad, i=1, \ldots, k ; j=1, \ldots, m$.

It is clear that we can find the $T_{i}$ with the desired properties. Since $\mathcal{F}$ is orientable and since we may assume that every component of every $T_{i}$ is a compact 2-manifold with non-empty boundary, tubular neighborhoods of the $T_{i}$ and $D_{i}$ are trivial. We also may assume that all fibres of these neighborhoods are open disks in leaves of $\mathcal{F}$. We find the desired transverse manifold $S$ by replacing each $T_{i}$ by $m_{i}=\left(\begin{array}{lll}n_{1} \cdot & \ldots & \cdot n_{k+m}\end{array}\right) / n_{i}$ disjoint copies of $T_{i}$ each being a section of the tubular neighborhood of $T_{i}$ and similarly $D_{i}$ by $\left(n_{1} \cdot \cdots \cdot n_{k+m}\right) / n_{k+i}$ copies, making sure that all these copies are disjoint. Then $S$ is the union of all these copies and $N=U_{k+1} \cup \cdots \cup U_{k+m}$.

\section{Appendix: the topological case}

The hypothesis that $\mathcal{F}$ is $C^{1}$ was used in the preceding section in two instances. First in the proof of 4.1 and second in the statement that exceptional leaves of a Seifert fibration are isolated. The first instance can be dealt with as in the proof of Theorem 3.3 in [Vo 2]. Instead of showing that each $D_{m} \backslash D_{m-1}$ is closed one shows that any component $R$ of $D_{m}$ which meets $D_{m-1}$ is entirely contained in $D_{m-1}$. This is Lemma 3.5 in [Vo 2] and its proof can be used in our situation since non-compact leaves do not figure in $D_{m}$.

Exceptional leaves need not be isolated in topological Seifert fibrations. See Remark 4.5 in [Vo 2]. There are two ways to get around this problem. One is to show that nevertheless we can argue as before in the proof of Claim 4.5 by showing the existence of compact invariant neighborhoods $U$ of these leaves with the following property: there is an invariant neighborhood $F$ of $F r_{B_{\alpha-1}} U$ in $B_{\alpha-1}$ such that all leaves in $F$ will intersect a transverse 2-manifold $D$ 
in the same number of points. Here $D$ is supposed to meet every leaf of $U$ and $U \cap \partial D=\emptyset$. The proof of the existence of such a $U$ follows from the arguments at the beginning of the proof of 4.5 where we decomposed $K_{0}$ into $K_{0,1} \cup \cdots \cup K_{0, k}$. Decompose $U$ by the same process into $U_{1} \cup \cdots U_{r}$ and then replace $U$ by the $U_{i}$ containing the exceptional leaf. This will have the required properties.

Another way to proceed is the use of the so called fine Epstein hierarchy instead of our version. Here $B_{\alpha+1}$ is defined to be the union of leaves $L$ in $B_{\alpha}$ such that for any open transverse manifold $T$ intersecting $L$ there are leaves of $B_{\alpha}$ intersecting $T$ in more than one point. Then $\mathcal{F}$ restricted to $B_{\alpha} \backslash\left(N_{\alpha} \cup B_{\alpha+1}\right)$ will be a locally trivial bundle and the problem of exceptional leaves disappears altogether.

\section{The foliation cycle and the proof of Theorem $2^{\prime}$}

We begin with a proof of the analogue of what is called the "Moving Leaf Proposition" in [EMS].

Proposition 5.1 Let $\mathcal{F}$ be a foliation of codimension $k$ on a manifold $M$, let $B_{1}$ be the first bad set of $\mathcal{F}$ and let $N_{0}$ be the union of all non-compact leaves of $\mathcal{F}$ in the complement of $B_{1}$. Assume that $N_{0} \cup B_{1}$ is not empty, that the closure of every leaf of $\mathcal{F}$ is compact and that one of the following two conditions holds

(i) $N_{0}$ is not open, or

(ii) $N_{0}=\emptyset$, $\operatorname{int} B_{1}=\emptyset$, and $B_{1} \backslash B_{2} \neq \emptyset$.

Then for any transverse $k$-manifold $T$ whose interior intersects every leaf of $B_{1}$ there exists a component $U$ of $M \backslash\left(N_{0} \cup B_{1}\right)$ such that $\sec _{T}$ is unbounded on $U$ (for notations see 3.1 and 3.2).

Proof Assume that (i) holds. Since $B_{1}$ is closed we find $x_{0} \in N_{0} \cap \operatorname{Fr} N_{0}$, where $F r N_{0}$ is the set theoretic boundary of $N_{0}$, and a neighborhood $V$ of $x_{0}$ with $V \cap B_{1}=\emptyset$. Let $y_{0}$ be a point of $V \backslash N_{0}$ and $U$ be the component of $M \backslash\left(N_{0} \cup B_{1}\right)$ containing $y_{0}$. Then $\sec _{T}$ will be unbounded on $\mathrm{U}$ for any transverse $k$-manifold $T$ such that int $T$ intersects every leaf of $B_{1}$. To see this let $x_{1}$ be a point in $(F r U) \cap V$. Since $N_{0} \cup B_{1}$ is closed $x_{1}$ lies in $N_{0}$. Since the closure of the leaf $L_{x_{1}}$ through $x_{1}$ is compact (by hypothesis) there exists a 
leaf $L \subset B_{1}$ in the limit set of $L_{x_{1}}$. Therefore $L_{x_{1}}$ will intersect any transverse manifold $T$ in infinitely many points if Int $T \cap L \neq \emptyset$. Since $L_{x_{1}} \subset F r U$ the function $\sec _{T}$ will be unbounded on $U$ by Property 3.3(a) of $\sec _{T}$.

If (ii) holds we distinguish two cases.

Case 1 All leaves of $B_{1} \backslash B_{2}$ are non-compact. Since int $B_{1}=\emptyset$, the union $N_{1}=B_{1} \backslash B_{2}$ of all non-compact leaves in the complement of $B_{2}$ is not open unless it is empty. By hypothesis, $B_{1}=B_{1} \cup N_{0}$ and $N_{1}=B_{1} \backslash B_{2}$ are not empty. Now, we can argue as before, replacing $N_{0}$ by $N_{1}$, and $B_{1}$ by $B_{2}$. In this way we find a component $U$ of $M \backslash\left(N_{1} \cup B_{2}\right)=M \backslash\left(N_{0} \cup B_{1}\right)$ which has the following property: $\sec _{T}$ is unbounded on $U$ for any transverse $k$-manifold $T$ whose interior intersects every leaf of $B_{2}$.

Case $2 \quad B_{1} \backslash B_{2}$ contains compact leaves. Since the union $N_{1}$ of all noncompact leaves of $B_{1} \backslash B_{2}$ is a closed subset of $M \backslash B_{2}$ the space $M \backslash\left(N_{1} \cup B_{2}\right)$ is a manifold and the restriction $\mathcal{F}_{1}$ of $\mathcal{F}$ to $M \backslash\left(N_{1} \cup B_{2}\right)$ is a foliation with all leaves compact. Furthermore, the first bad set of $\mathcal{F}_{1}$ is $B_{1} \backslash\left(B_{2} \cup N_{1}\right)$ and therefore not empty.

The Moving Leaf Proposition in [EMS] requires the bad set to be compact and $B_{1} \backslash\left(B_{2} \cup N_{1}\right)$ need not be compact. Now there are two parts in the proof of the Moving Leaf Proposition in [EMS]. The first (and most difficult) part states that there is a component of the complement of the first bad set on which the volume of leaf function is not bounded. The proof does not make any use of the compactness of $B_{1}\left(\mathcal{F}_{1}\right)$. It is purely local. In fact what is proved in [EMS] in the two paragraphs starting with the last paragraph on page 23 can be stated as follows: Let $\mathcal{G}$ be a foliation of codimension $k$ with all leaves compact and $L$ any leaf in $B_{1}(\mathcal{G})$ such that $L$ has trivial holonomy in the foliated set $B_{1}(\mathcal{G})$. Let $D$ be any transverse $k$-disk intersecting $L$ in its interior $\stackrel{\circ}{D}$. Then there exists a component $V$ of $\stackrel{\circ}{D} \backslash B_{1}(\mathcal{G})$ such that $\sec _{D}$ is unbounded on $V$.

Since the union of leaves of $B_{1}(\mathcal{G})$ with trivial holonomy in $B_{1}(\mathcal{G})$ is open and dense, our claim is an immediate consequence of the above statement when applied to $\mathcal{G}=\mathcal{F}_{1}$.

Next we will construct a particular foliation cycle. This is the point where compactness of $N_{0} \cup B_{1}$ is essential. Compactness of $N_{0} \cup B_{1}$ guarantees the existence of arbitrarily small saturated compact neighborhoods $X$ of $N_{0} \cup B_{1}$. This is due to the fact that the frontier $\operatorname{Fr}(W)$ of a relatively compact neighborhood $W$ of $N_{0} \cup B_{1}$ is a compact subset of $M \backslash\left(N_{0} \cup B_{1}\right)$, and on $M \backslash\left(N_{0} \cup B_{1}\right)$ the foliation $\mathcal{F}$ is a Seifert fibration. Therefore, the saturation $S$ of $\operatorname{Fr}(W)$ is 
also compact and thus closed. Then $Y=W \backslash S$ is a saturated neighborhood of $N_{0} \cup B_{1}$ with $C l(Y) \subset C l(W)$.

From now on we will assume that $N_{0} \cup B_{1}$ is compact and non-empty, that $N_{0}$ is either empty or not open und that $\bigcap_{\alpha} B_{\alpha}=\emptyset$. Furthermore, we assume that $\mathcal{F}$ is $C^{1}$ and oriented. The last condition allows us to consider the compact leaves of $\mathcal{F}$ as $(n-k)$-dimensional cycles.

Let $X$ be a compact saturated neighborhood of $N_{0} \cup B_{1}$. Then we can find finitely many foliation charts $W_{i}=E_{i} \times T_{i}, i=1, \ldots, s$, whose interiors cover $X$. Here we assume that each $T_{i}$ is a compact transverse $k$-manifold and each $E_{i} \times\{t\}$ is an open relatively compact subset of a leaf. As usual, we assume that each $E_{i} \times T_{i}$ is part of a larger foliation chart $\tilde{E}_{i} \times \tilde{T}_{i}$ with $T_{i} \subset \operatorname{int} \tilde{T}_{i}$ and $C l\left(E_{i}\right) \subset$ int $\tilde{E}_{i}$. We may and will assume that the $T_{i}$ are disjoint. Then $T=\bigcup T_{i}$ is a compact transverse $k$-manifold whose interior int $T=\bigcup$ int $T_{i}$ intersects every leaf of $B_{1}$.

Let $U$ be a component of $X \backslash\left(B_{1} \cup N_{0}\right)$ such that $\sec _{T}$ is unbounded on $U$. By Propositions 5.1 and 3.7 such a component exists. Let $L_{1}, L_{2}, \ldots$ be a sequence of leaves in $U$ such that $\sec _{T}\left(L_{i}\right)$ is a strictly increasing unbounded sequence. Since $\sec _{T}$ is bounded on any compact subset of $U$ the sequence of leaves $L_{1}, L_{2}, \ldots$ converges to $B_{1} \cup N_{0}$. Since the union of all leaves of $U$ with trivial holonomy is open and dense we may and will assume that the leaves $L_{i}$ have trivial holonomy. Then all leaves $L_{i}$ are homologous in $U$.

In $\S \S 2$ and 3 of [EMS] is explained how this set-up leads after passing to a suitable subsequence and the appropriate choice of integers $n_{i}$ to a limiting foliation cycle $\lim _{i} \frac{1}{n_{i}} L_{i}$. This foliation cycle will be essential in the proof of Theorem $2^{\prime}$. We repeat its construction. For each $i$ let $n_{i}=\max \left\{\sec _{T_{j}}\left(L_{i}\right)\right.$ : $j=1, \ldots, s\}$. By passing to a subsequence of the $L_{i}$ and reordering the $T_{j}$ we may assume that $n_{i}=\sec _{T_{1}}\left(L_{i}\right)$. We define a non-negative measure $\mu_{j, i}$ on the Borel sets of $T_{j}$ by assigning each point of $T_{j} \cap L_{i}$ the mass $\frac{1}{n_{i}}$. Then $\mu_{j, i}\left(T_{j}\right) \leq 1$ for all $i, j$ and $\mu_{1, i}\left(T_{1}\right)=1$ for all $i$. Consequently, after passing to a further subsequence of the $L_{i}$, we may assume that for all $j$ the measures $\mu_{j, i}$ converge to a non-negative measure $\mu_{j}$ on $T_{j}$ with $\mu_{j}\left(T_{j}\right) \leq 1$ and $\mu_{1}\left(T_{1}\right)=1$.

By Lemma A of $\S 3$ of [EMS] the measures $\left\{\mu_{j}\right\}$ are holonomy invariant and therefore define a geometric current $C\left\{\mu_{j}\right\}$. The associated closed de Rham current is equal to $\lim _{i} \frac{1}{n_{i}} L_{i}$, i. e. for any $(n-k)$-form $\omega$ defined in a neighborhood of $X$ we have $\left\langle C\left\{\mu_{j}\right\}, \omega\right\rangle=\lim _{i} \frac{1}{n_{i}} \int_{L_{i}} \omega$. This is Lemma B of [EMS]. From this we obtain the first important property of our foliation cycle $C\left\{\mu_{j}\right\}$. 
5.2 (Property 1 of the foliation cycle $C\left\{\mu_{j}\right\}$ ) Let $\omega$ be any closed $(n-k)-$ form defined in $\operatorname{int} X$ then $\left\langle C\left\{\mu_{j}\right\}, \omega\right\rangle=0$.

Proof This is due to the simple fact that the leaves $L_{1}, L_{2}, \ldots$ are all homologuous in $\operatorname{int} U \subset \operatorname{int} X$ so that the sequence $\int_{L_{i}} \omega$ is constant if $\omega$ is closed. Since $1 / n_{i}$ converges to 0 we are done.

The proof of Theorem $2^{\prime}$ is now an immediate consequence of the second property of $C\left\{\mu_{j}\right\}$.

5.3 (Property 2 of the foliation cycle $C\left\{\mu_{j}\right\}$ ) Let $\omega$ be any closed $(n-k)$ form defined in a neighborhood of $X$ such that for any compact leaf $L$ of $B_{1}$ the inequality $\int_{L} \omega>0$ holds. Then

$$
\left\langle C\left\{\mu_{j}\right\}, \omega\right\rangle>0 \text {. }
$$

Proof Recall the definition of the de Rham current

$$
\left\langle C\left\{\mu_{j}\right\},-\right\rangle: \Omega^{n-k}\left(M_{0}\right) \longrightarrow \mathbb{R},
$$

where $M_{0}$ is any neighborhood of $X$. One chooses a partition of unity $p_{1}, \ldots, p_{s}$ subordinate to the covering of $X$ by the interiors of $W_{j}=E_{j} \times T_{j}$ and defines for any $\eta \in \Omega^{n-k}\left(M_{0}\right)$

$$
\left\langle C\left\{\mu_{j}\right\}, \eta\right\rangle=\sum_{j} \int_{T_{j}}\left(\int_{E_{j} \times\{t\}}\left(p_{j} \cdot \eta\right)\right) d \mu_{j}(t) .
$$

The definition is easily seen to be independent of the choice of partition of unity. [EMS], $\S 2$.

We need to change the "local" recipe for calculating $\left\langle C\left\{\mu_{j}\right\}, \eta\right\rangle$ to a more global one where we integrate $(n-k)$-forms over total leaves instead of plaques $E_{j} \times\{t\}$.

First we notice that for every $j$ the measure $\mu_{j}$ is supported on $T_{j} \cap B_{1}$. Clearly, $\mu_{j}$ is supported on $\left(N_{0} \cup B_{1}\right) \cap T_{j}$ since the sequence $L_{i}$ converges to the closed set $N_{0} \cup B_{1}$. Let $x$ be a point of $N_{0} \cap T_{j}$ and $\tilde{T}_{j}$ a transverse $k$-manifold such that $T_{j} \subset$ Int $\tilde{T}_{j}$. Then we find a transverse $k$-manifold $D \subset \operatorname{int} \tilde{T}_{j}$ such that $x \in \operatorname{int} D$ and $\sec _{D}$ is bounded. In particular, the number of intersection points of $L_{i}$ with $D$ is bounded, and this implies that $\mu_{j}(D)=0$.

By hypothesis $\bigcap_{\alpha} B_{\alpha}=\emptyset$. Then Proposition 3.6 tells us that $B_{1}$ is a countable disjoint union of Borel sets: 


$$
B_{1}=\bigcup_{\alpha \geq 1}\left(B_{\alpha} \backslash B_{\alpha+1}\right)
$$

As before, denote the union of all non-compact leaves of $B_{\alpha} \backslash B_{\alpha+1}$ by $N_{\alpha}$. Then we make the following claim.

Claim 5.4 For all $\alpha$ and $j$ the equation $\mu_{j}\left(N_{\alpha} \cap T_{j}\right)=0$ holds.

Proof of Claim We have already proved this statement for $\alpha=0$ using an easy argument. In outline, the statement is true in general because leaves of $N_{\alpha}$ have their limit points in $B_{\alpha+1}$, and, if $B_{\alpha+1} \cup N_{\alpha}$ is compact, their limit sets are non-empty. If $\mu_{j}\left(N_{\alpha} \cap T_{j}\right)>0$ for some $j$, we find a compact set $E \subset N_{\alpha} \cap T_{j}$ in the complement of $B_{\alpha+1}$ with $\mu_{j}(E)>0$. Using holonomy translations repeatedly we can push $E$ into a countable disjoint family of subsets in $\bigcup_{i} T_{i}$. The holonomy invariance of the measures then implies that each of these sets has measure not less than $\mu_{j}(E)$. This will contradict the fact that for all $i$ we have $\mu_{i}\left(T_{i}\right) \leq 1$.

In more detail, assume that $\mu_{j}\left(N_{\alpha} \cap T_{j}\right)>0$. Then by passing, if necessary, to a different $T_{j}$ we may also assume that $\mu_{j}\left(N_{\alpha} \cap \operatorname{int} T_{j}\right)>0$. The set $N_{\alpha} \cap \operatorname{int} T_{j}$ is covered by (countably many) sets of the form $N_{\alpha} \cap S$ such that $S$ is open $\operatorname{in} \operatorname{int} T_{j}$ and $\sec _{S}$ is bounded on $N_{\alpha}$. So we may assume that $\mu_{j}\left(S \cap K_{0}\right)>0$ for some such $S$ and some compact subset $K_{0}$ of the closed subset $S \cap N_{\alpha}$ of $S$. By (3.3) $\sec _{S}$ is lower semicontinuous and thus by the Baire category theorem there exists an open dense subset of $K_{0}$ where $\sec _{S}$ is continuous. Let $K_{1}$ be its complement in $K_{0}$. Then clearly $\max \left\{\sec _{S}(x) \mid x \in K_{1}\right\}<$ $\max \left\{\sec _{S}(x) \mid x \in K_{0}\right\}$. Continuing inductively we find a finite sequence $K_{0} \supset K_{1} \supset K_{2} \supset \cdots \supset K_{r}=\varnothing$ such that $\sec _{S}$ is locally constant on $K_{i} \backslash K_{i+1}$ for all $i \geq 0$. Using this, we find an open subset $U$ of $T_{j}$ and a compact subset $N$ of $U \cap N_{\alpha}$ with $\mu_{j}(N)>0$ such that $\sec _{U}(x)=1$ for all $x \in N$.

Now, each leaf through a point of $N$ accumulates against $B_{\alpha+1}$. Using holonomy translations along the leaf through $x \in N$ we find a curve $\omega$ through this leaf and a neighborhood $V(x)$ of $x$ in $N$ such that $\omega$ holonomy-translates $V(x)$ into a set, $V^{\prime}(x)$, contained in some $T_{i}$ such that $N \cap V^{\prime}(x)=\varnothing$. By compactness of $N$ finitely many of such $V(x)$ suffice to cover $N$. Assuming that each $V(x)$ is compact the union of these $V^{\prime}(x)$ constitute a compact set $N^{\prime}$ disjoint from $N$ such that $\sum_{i} \mu_{i}\left(N^{\prime} \cap T_{i}\right) \geq \mu_{j}\left(N \cap T_{j}\right)$. The inequality is due to the fact that $\sum \mu_{i}$ is holonomy invariant and that each leaf of $N_{\alpha}$ intersects $N$ in at most one point. Therefore, if $V^{\prime}\left(x_{1}\right)$ and $V^{\prime}\left(x_{2}\right)$ intersect, then $V\left(x_{1}\right)$ and $V\left(x_{2}\right)$ intersect in a set of at least the same measure. The 
inequality then follows by induction on the number of $V\left(x_{i}\right)$ used to cover $N$. Since $N^{\prime}$ is compact, we can do the process over again, moving open sets $W(x)$ of $N$ to sets $V^{\prime \prime}(x)$ in some $T_{i}$ such that $V^{\prime \prime}(x) \cap\left(N \cup N^{\prime}\right)=\varnothing$, obtaining a set $N^{\prime \prime}$ such that $\sum_{i} \mu_{i}\left(N^{\prime \prime} \cap T_{i}\right) \geq \mu_{j}(N)$. Continuing, we find compact subsets of $\bigcup_{i}\left(N_{\alpha} \cap T_{i}\right)$ of arbitrary large measures contradicting $\mu_{i}\left(T_{i}\right) \leq 1$ for all $i$.

Therefore, for any $j$

$$
\mu_{j}\left(T_{j}\right)=\sum_{\alpha \geq 1} \mu_{j}\left[\left(B_{\alpha} \backslash\left(B_{\alpha+1} \cup N_{\alpha}\right)\right) \cap T_{j}\right] .
$$

If $\mu_{\alpha, j}$ denotes the restriction of $\mu_{j}$ to $\left(B_{\alpha} \backslash\left(B_{\alpha+1} \cup N_{\alpha}\right)\right) \cap T_{j}$, then $\left\{\mu_{\alpha, j}\right\}, j=$ $1, \ldots, s$, defines for any $\alpha \geq 1$ a holonomy invariant transverse measure on $T=\bigcup_{j} T_{j}$. We denote by $C\left\{\mu_{\alpha, j}\right\}$ the associated foliation cycle. Then

$$
C\left\{\mu_{j}\right\}=\sum_{\alpha \geq 1} C\left\{\mu_{\alpha, j}\right\}
$$

The proof of (5.3) is thus a consequence of the next lemma.

Lemma 5.5 Let $\omega$ be any $(n-k)$-form defined in a neighborhood of $B_{1}$ such that $\int_{L} \omega>0$ for any compact leaf of $B_{1}$. Then for any $\alpha \geq 1$ we have $\left\langle C\left\{\mu_{\alpha, j}\right\}, \omega\right\rangle \geq 0$ and there exists at least one $\alpha \geq 1$ such that $\left\langle C\left\{\mu_{\alpha, j}\right\}, \omega\right\rangle>0$.

Proof A proof of this lemma can easily be extracted from $\S \S 6$ and 7 in [EMS]. Our set-up is slightly different. In particular, we use what in [EMS] is called the coarse Epstein filtration. For the convenience of the reader we give a direct proof of 5.5 adjusted to our situation. Fix $\alpha \geq 1$. The foliation $\mathcal{F}$ restricted to $S_{\alpha}=B_{\alpha} \backslash\left(B_{\alpha+1} \cup N_{\alpha}\right)$ is a foliation with all leaves compact. By definition of the Epstein hierarchy (see 3.4) there exists for any leaf $L$ of $S_{\alpha}$ a transverse disk $D$ such that $L$ intersects int $D$ and $\sec _{D}$ is bounded on $S_{\alpha}$. This implies that $\mathcal{F}$ restricted to $S_{\alpha}$ is a Seifert fibration which translates into a very explicit description of a foliated neighborhood $U_{L}$ of $L$ in $S_{\alpha}$ as follows (for details see [Ep 2]). Let $D$ be a transverse manifold whose interior intersects $L$. Let $x$ be a point in $\operatorname{int} D \cap L$. Then we find a neighborhood $U_{x}$ of $x$ in $S_{\alpha} \cap D$, a finite group $H$ of homeomorphisms of $U_{x}$ fixing $x$, and a finite regular covering $\tilde{L} \longrightarrow L$ with deck transformation group isomorphic to $H$ such that as a foliated set $U_{L}$ is isomorphic to $\left(\tilde{L} \times U_{x}\right) / H$. Here $H$ operates diagonally on $\tilde{L} \times U_{x}$ and $\left(\tilde{L} \times U_{x}\right) / H$ is foliated by the images of $\tilde{L} \times\{t\}, t \in U_{x}$. By choosing $U_{x}$ 
sufficiently small we may assume that for any $h \in H$ the germ of $h$ at $x$ is not trivial, so that $H$ realizes the holonomy group of $L$ in the foliated set $S_{\alpha}$.

Fixing again a leaf $L$ of $S_{\alpha}$ we find an index $j_{0}$ such that $L \cap \operatorname{int} T_{j_{0}} \neq \emptyset$. Then we may choose $x \in L \cap \operatorname{int} T_{j_{0}}$ and $U_{x} \subset \operatorname{int} T_{j_{0}}$ in the above discussion to have the following additional properties:

Property 5.6 For any $j \in\{1, \ldots, s\}$ and $y \in L \cap \operatorname{int} T_{j}$ there exists a holonomy translation

$$
h_{y x}: U_{x} \longrightarrow \operatorname{int} T_{j}
$$

along a path in $L$ from $x$ to $y$ such that

(i) $h_{y x}\left(U_{x}\right) \cap h_{y^{\prime} x}\left(U_{x}\right)=\emptyset$, if $y \neq y^{\prime}$;

(ii) Let $\left\{p_{j}\right\}$ be the partition of unity subordinate to $\left\{\operatorname{int} W_{j}=E_{j} \times \operatorname{int} T_{j}\right\}$ used in our formula for evaluating our foliation cycle on forms. Then for every $j$ the projection to $\operatorname{int} T_{j}$ of the intersection of the neighborhood $U_{L}=\left(\tilde{L} \times U_{x}\right) / H$ of $L$ with $\operatorname{supp}\left(p_{j}\right) \subset E_{j} \times \operatorname{int} T_{j}$ is contained in $\bigcup_{y \in \operatorname{int} T_{j}} h_{y x}\left(U_{x}\right)$.

Using these properties we can rewrite the contribution of $U_{L}$ to $C\left\{\mu_{\alpha, j}\right\}$ :

$$
\begin{aligned}
& \sum_{j} \int_{T_{j} \cap U_{L}}\left(\int_{E_{j} \times\{t\}} p_{j} \cdot \omega\right) d \mu_{\alpha, j}(t)= \\
= & \sum_{j} \sum_{y \in L \cap i n t T_{j}} \int_{U_{x}}\left(\int_{E_{j} \times\left\{h_{y x}(t)\right\}} p_{j} \cdot \omega\right) d \mu_{\alpha, j_{0}}(t)= \\
= & \int_{U_{x}}\left(\sum_{j} \sum_{y \in L \cap \operatorname{int} T_{j}} \int_{E_{j} \times\left\{h_{y x}(t)\right\}} p_{j} \cdot \omega\right) d \mu_{\alpha, j_{0}}(t) .
\end{aligned}
$$

The first equation is a consequence of the holonomy invariance of $\left\{\mu_{\alpha, j}\right\}$ and property 5.6. The second equation is obvious.

Also by holonomy invariance the last expression does not change if $t \in U_{x}$ is replaced by $h(t)$ with $h \in H$. Therefore, it is equal to

$$
\frac{1}{|H|} \int_{U_{x}}\left(\sum_{j} \sum_{y \in L \cap i n t T_{j}} \sum_{h \in H} \int_{E_{j} \times\left\{h_{y x} \circ h(t)\right\}} p_{j} \cdot \omega\right) d \mu_{\alpha, j_{0}}(t) .
$$

Using once more (5.6) we see that for any $t \in U_{x}$ 


$$
\sum_{y \in L \cap \operatorname{int} T_{j}} \sum_{h \in H} \int_{E_{j} \times\left\{h_{y x} \circ h(t)\right\}} p_{j} \cdot \omega=\left|H_{t}\right| \cdot \int_{L_{t}} p_{j} \cdot \omega
$$

where $L_{t}$ denotes the leaf through $t$, and $H_{t}$ is the stabilizer of $t$ in $H$. Altogether we see that the contribution of $U_{L} \subset S_{\alpha}$ to $C\left\{\mu_{\alpha, j}\right\}$ when evaluated on the form $\omega$ equals

\section{7}

$$
\frac{1}{|H|} \int_{U_{x}}\left(\left|H_{t}\right| \int_{L_{t}} \omega\right) d \mu_{\alpha, j_{0}(t)} .
$$

Exactly the same formula holds when $U_{x}$ is replaced by an $H$-invariant measurable subset $\tilde{U}$ of $U_{x}$ and $U_{L}$ by the union $\tilde{U}_{L}$ of leaves through $\tilde{U}$. Now, $S_{\alpha}$ is a countable disjoint union of sets of the form $\tilde{U}_{L}$. To see this cover $S_{\alpha}$ by a locally finite (and therefore countable) family of open Seifert fibred neighborhoods $U_{L_{k}}$ of leaves $L_{k}$ having all the properties needed for the discussion above and having compact closure in $S_{\alpha}$. Then $S_{\alpha}$ is the disjoint union of $U_{L_{k}} \backslash \bigcup_{i<k} U_{L_{i}}, k=1,2, \ldots$.

The proof of Lemma 5.5 is now immediate from (5.7). The hypothesis that for any compact leaf $L \subset B_{1}$ the integral $\int_{L} \omega$ is positive guarantees that the expression in (5.7) is never negative. On the other hand, for some $\alpha \geq 1$ and some $j_{0}$ the measure $\mu_{\alpha, j_{0}}\left(\operatorname{int} T_{j_{0}}\right)=\mu_{j_{0}}\left(\operatorname{int} T_{j_{0}} \cap S_{\alpha}\right)$ is positive since $\left\{\mu_{j}\right\}$ is holonomy invariant, $\mu_{1}\left(T_{1}\right)=1$ and $\left\{\operatorname{int} T_{j} \times E_{j}\right\}$ covers all of $B_{1} \cup N_{0}$. Finally $t \longmapsto\left|H_{t}\right| \int_{L_{t}} \omega$ is continuous on $U_{x}$ and everywhere positive.

\section{References}

[EMS] R Edwards, K C Millett, D Sullivan, Foliations with all Leaves Compact, Topology 16 (1977) 13-32

[Ep 1] D B A Epstein, Periodic flows on 3-manifolds, Annals of Math. 95 (1972) 58-82

[Ep 2] D B A Epstein, Foliations with all leaves compact, Annales de l'Institut Fourier Grenoble 26 (1976) 265-282

[Ep 3] D B A Epstein, Pointwise Periodic Homeomorphisms, Proc. London Math. Soc., 3. Ser.42 (1981) 415-460

[EMT] D B A Epstein, K C Millett, D Tischler, Leaves without holonomy, J. London Math. Soc. (2) 16 (1977) 548-552 
[Hae] A Haefliger, Variétés feuilletées. Ann. Scuola Norm. Sup. Pisa 16 (1962) 367-397

[Ku] K Kuratowski, Topology Vol 2 Academic Press 1968

[L] R Langevin, A list of questions about foliations, in: Differential Topology, Foliations, and Group Actions, P Schweitzer et al (editors), Contemporary Mathematics 161 Amer. Math. Soc. (1994) 59-80

[M] D Montgomery, Pointwise periodic homeomorphisms, Amer. J. Math. 59 (1937) 118-120

[R] G Reeb, Sur certaines propriétés topologiques des variétés feuilletées, Act. Sci. Indust., Hermann 1952

[Vo 1] E Vogt, Foliations of Codimension 2 with all Leaves Compact, manuscripta math. 18 (1976) 187-212

[Vo 2] E Vogt, Bad sets of compact foliations of codimension 2, Proceedings of Low Dimensional Topology (K. Johannson ed.) Knoxville 1992, International Press Company (1994) 187-216

[Vo 3] E Vogt, Negative Euler characteristic as an obstruction to the existence of periodic flows on open 3-manifolds, in: T Mizutani et al (eds.), Proceedings of the international symposium/workshop on "Geometric Study of Foliations", World Scientific (1994) 457-474

[Wea] N Weaver, Pointwise periodic homeomorphisms, Annals of Math. 95 (1972) $83-85$

2, Mathematisches Institut, Freie Universität Berlin Arnimallee 3, 14195 Berlin, Germany

Email: vogt@math.fu-berlin.de

Received: 23 July 2001 Revised: 3 April 2002 